\title{
A randomized phase II non-comparative study of pemetrexed-carboplatin and gemcitabine-vinorelbine in anthracycline- and taxane-pretreated advanced breast cancer patients
}

\author{
DINO AMADORI ${ }^{1}$, EVA CARRASCO $^{2}$, SIEGFRIED ROESEL $^{3}$, ROBERTO LABIANCA $^{4}$, BEATRICE UZIELY $^{5}$, \\ VICTORIA SOLDATENKOVA ${ }^{6}$, VALERIE MOREAU ${ }^{7}$, DURISALA DESAIAH ${ }^{8}$, \\ THOMAS BAUKNECHT ${ }^{6}$ and MIGUEL MARTIN ${ }^{9}$ \\ ${ }^{1}$ Romagnolo Scientific Institute for the Study and Treatment of Cancer, Meldola, Italy; ${ }^{2}$ Spanish Breast Cancer \\ Research Group (GEICAM), Madrid, Spain; ${ }^{3}$ Gütersloh Oncology Practice, Gütersloh, Germany; \\ ${ }^{4}$ Riuniti Hospital, Bergamo, Italy; ${ }^{5}$ Hadassah-Hebrew University Medical Center, Jerusalem, Israel; \\ ${ }^{6}$ Lilly Deutschland, Bad Homburg, Germany; ${ }^{7}$ Lilly France, Paris, France; ${ }^{8}$ Eli Lilly and Company, \\ Indianapolis, IN, USA; ${ }^{9}$ Gregorio Marañón University Hospital Research Institute, \\ Complutense University, Madrid, Spain
}

Received September 19, 2012; Accepted November 12, 2012

DOI: $10.3892 /$ ijo.2013.1869

\begin{abstract}
Pemetrexed-carboplatin and gemcitabine-vinorelbine combination therapies were efficacious in phase II and phase III studies as first-line breast cancer treatment. Thus, Arm A and Arm B combinations were investigated in patients pretreated with anthracycline and taxanes. Women with advanced breast cancer, with $\geq 1$ measurable lesion per RECIST, were stratified by line of treatment (1st, 2nd), visceral disease (yes/no), ECOG PS (0-1 vs. 2) and randomized 1:1 to Arm A (pemetrexed 600 mg/m², D1 i.v.q21; carboplatin, AUC 5, D1 i.v.q21) or Arm B (gemcitabine 1,200 mg/m² D1, D8 i.v. q21; vinorelbine $30 \mathrm{mg} / \mathrm{m}^{2}$ D1, D8 i.v. q21). Treatment continued until progression. The primary endpoint was objective response rate (RR). Secondary endpoints were duration of response (DoR), time-to-response (TTR), time-to-progressive disease (TTPD), time-to-treatment failure (TTTF) and safety. A two-stage design was employed independently for each arm. Of 135 randomized patients, 125 (Arm A, n=64; Arm B, n=61) qualified for tumor-response analysis. The mean (standard deviation) number of cycles administered was 6.3 (4.13) in Arm A and 6.2 (4.39) in Arm B. Efficacy in Arm A and Arm B were: RR (95\% CI), 26.6 (16.339.1) and 29.5 (18.5-42.6); time-to-events (months), DoR 7.7 and
\end{abstract}

Correspondence to: Professor Dino Amadori, Romagnolo Scientific Institute for the Study and Treatment of Cancer (I.R.S.T.) S.r.l., Via Piero Maroncelli 40, I-47014 Meldola, Italy

E-mail: direzione.scientifica@irst.emr.it

Key words: advanced breast cancer patients, combination therapy, pemetrexed-carboplatin and gemcitabine-vinorelbine, response rates, safety and tolerability
7.5; TTPD, 5.1 and 5.6; TTR, 1.8 and 1.8; TTTF, 4.8 and 5.1; respectively. Most common grade $3 / 4$ adverse events possibly related to study-drug were neutropenia, thrombocytopenia, anemia and leucopenia in Arm A and neutropenia, leucopenia and fatigue in Arm B. In this study, both combinations showed moderate activity as predefined RR was not reached and were well tolerated.

\section{Introduction}

Breast cancer is the most frequently diagnosed cancer, second only to lung cancer, and is the leading cause of cancer death in women worldwide, accounting for $23 \%$ (1.38 million) of the total new cancer cases and $14 \%(458,400)$ of the total cancer deaths in $2008(1,2)$. Breast cancer continues to have a $20 \%$ rate for advanced or metastatic disease with a palliative prognosis, although it has achieved higher cure rates because of earlier detection and the likelihood that better treatments have led to lower mortality rates (1). However, the optimization of treatment procedures in metastatic disease remains an unmet need.

A number of cytotoxic drugs have substantial antitumor activity against breast cancer. Combinations of cytotoxic regimens are associated with higher response rates (RR) and longer durations of response than single-agent regimens (3). However, the survival rate is very low for the patients who ultimately develop metastatic disease (4). Thus, improved therapies for patients with advanced stages of the disease are necessary. Many compounds have shown activity in metastatic breast cancer; however, there is a need for well-tolerated active combinations, particularly in high-risk groups, e.g., patients with visceral metastasis having a fast progression rate $(5,6)$.

Pemetrexed, as a single agent, in anthracycline- and taxanepretreated breast cancer patients has shown a RR of 9-28\% (7-9). 
Platinum compounds are being increasingly incorporated in the treatment of metastatic breast cancer (10-13) as they have a synergistic action with pemetrexed and gemcitabine (14). Carboplatin, with a similar activity to other platinum compounds, is a possible combination partner with pemetrexed, due to its better tolerability and safety profile $(13,15)$. Moreover, this combination has shown an overall response rate (ORR) of $54 \%$ and a manageable toxicity profile as a first-line therapy in patients with locally advanced and metastatic breast cancer (13).

Gemcitabine, as a single agent, has been studied in phase II trials (first-, second- and third-line) and a registration trial in combination with paclitaxel after anthracycline pretreatment received approval, reflecting its increasing acceptance as a standard treatment in first- and second-line metastatic breast cancer (16-22). After anthracycline and taxane pretreatment there has been an increasing list of trials with gemcitabine, both as a single agent but also in combinations $(14,17,21,23)$. It is known to have a better toxicity profile and non-overlapping toxicity with other chemotherapeutic agents and is thus advantageous for combination therapies $(24,25)$. The most promising of these was the phase III trial with gemcitabine and vinorelbine $(26,27)$.

Vinorelbine has been studied in phase II trials and has shown efficacy as a single agent in first- and second-line treatment after anthracycline pretreatment $(25,28,29)$. In other phase II studies, vinorelbine in combination with gemcitabine showed efficacy and tolerability as first-line therapy as well as after pretreatment either with anthracycline alone or with anthracycline/taxane-based regimens (30-33). A phase III trial with a vinorelbine/gemcitabine doublet in pretreated patients has also shown activity with an acceptable safety profile (27).

The pemetrexed-carboplatin combination has been tested $(13,15)$ and needs additional evaluation as a possible efficacious combination treatment after anthracycline and taxane pretreatment. The potential synergism between these compounds makes them an attractive combination to be compared to another well-tolerated combination in metastatic breast cancer disease such as gemcitabine-vinorelbine. The present study was conducted to elucidate the activity of two chemotherapy regimens in advanced breast cancer in a randomized phase II study. The primary objective was to determine the antitumor activity of pemetrexed-carboplatin and gemcitabinevinorelbine in anthracycline- and taxane-pretreated patients with advanced breast cancer, by measuring the RR [complete response $(\mathrm{CR})$ and partial response $(\mathrm{PR})]$. The secondary objective was to estimate time-to-event efficacy variables, safety and quality of life with both of those combination regimens.

\section{Materials and methods}

Patients. Adult females with a histologic or cytologic diagnosis of advanced breast cancer, who had received at least 1 prior chemotherapy containing anthracycline and taxanes, had at least 1 unidimensionally measurable lesion meeting the Response Evaluation Criteria in Solid Tumors (RECIST) (34), and had an Eastern Cooperative Oncology Group performance status (ECOG PS) of 0-2 with an estimated life expectancy of $\geq 3$ months were included in the study. Previous radiation therapy to less than $25 \%$ of the bone marrow was allowed, provided that the therapy was completed 30 days prior to study entry. Patients were not eligible if they had previously received pemetrexed, gemcitabine, carboplatin or vinorelbine, whether in clinical practice or in another clinical trial. Other exclusion criteria included the following: active infection; history of malignant conditions (except non-melanotic skin cancer or carcinoma in situ of the cervix); untreated cerebral metastases; inability or unwillingness to take folic acid, vitamin B12 supplementation or dexamethasone; or having received any investigational drug within 30 days of study entry.

The study was conducted according to the principles of good clinical practice, applicable laws and regulations, and the Declaration of Helsinki. Each institution's review board approved the study and all patients signed an informed consent document before study participation.

Study design. This was a multicenter, randomized (1:1), two-stage, open-label, non-comparative, parallel-group phase II study (NCT00325234) conducted between June 2006 and April 2010. Eligible patients with advanced breast cancer previously treated with anthracycline and taxanes were randomized either to Arm A (pemetrexed and carboplatin) or to Arm B (vinorelbine and gemcitabine). In Arm A, patients were administered with pemetrexed $600 \mathrm{mg} / \mathrm{m}^{2}$ (intravenously for $10 \mathrm{~min}$ on day 1) based on results from phase I study (35) and phase II study (13) and carboplatin [given over approximately $30 \mathrm{~min}$ beginning after the end of the pemetrexed infusion for target area under the curve (AUC) 5.0] on day 1, after pretreatment with folic acid, vitamin B12 and dexamethasone. In Arm B, vinorelbine $30 \mathrm{mg} / \mathrm{m}^{2}$ (given over approximately 6-10 $\mathrm{min}$ ) and gemcitabine $1,200 \mathrm{mg} / \mathrm{m}^{2}$ (given over approximately $30 \mathrm{~min}$ ) were administered on day 1 and day 8 . For treatment, a cycle was defined as an interval of 21 days. Patients were treated until unacceptable toxicity or progressive disease. Dose adjustments were based on the National Cancer Institute Common Toxicity Criteria (NCI-CTC) version 3.0 (36). Patients were stratified by line of treatment (first/second line), visceral disease (yes/no), and ECOG PS (0-1/2).

Efficacy and health outcome measures. The primary objective of this study was to assess antitumor activity independently for each of the arms, as measured by tumor RR (proportion of patients with CR or PR) according to RECIST (1.0). Radiological assessments were routinely performed before drug administration at every other cycle throughout the treatment. The secondary objectives included the assessment of time-to-event efficacy variables along with characterization of the quantitative and qualitative toxicities in each treatment arm in this patient population and assessment of quality of life. The time-to-event efficacy variables were duration of response (DoR) defined as the time from the date when the measurement criteria are met for complete response or partial response (whichever status is recorded first) until the date of first observation of disease progression or death from study disease, time-to-response (TTR) defined as the time from the date of study enrollment to the first date when the measurement criteria are met for complete response or partial response (whichever status is recorded first), time-to-progressive disease (TTPD) defined as the time from the date of study enrollment to the first documented date of progressive disease or death from study disease, and time-to-treatment failure (TTTF), defined as the time from date of study enrollment to the first documented date of death from any cause, progressive disease, or study treat- 
ment discontinuation due to adverse event. Quality of life (QoL) was assessed using the European Organization for Research and Treatment of Cancer (EORTC) questionnaires QLQ-C30 and QLQ-BR23 (37).

Safety measures. Patients who received at least 1 dose of study medication were evaluated for safety according to the following variables: extent of exposure; treatment-emergent adverse events [(TEAEs), graded using the Common Terminology Criteria for Adverse Events (CTCAE) scale, Version 3.0]; discontinuations due to adverse events (AEs); deaths; and serious adverse events (SAEs). All patients continued clinical follow-up visits for approximately 30 days after the last day of study-drug administration and every three months thereafter until disease progression, or for up to one year.

Statistical analysis. A two-stage design was employed independently for each of the arms, with the possibility of stopping each treatment early due to lack of response (38). For the first stage, 28 patients were to be evaluated per treatment arm. If fewer than or equal to 7 out of the 28 patients showed response to the investigational regimen, the accrual for this regimen was to be stopped and the conclusion was to be drawn that this regimen is not worthy of further study in this tumor type. If more than 7 patients showed response, accrual was to be continued until 68 qualified patients had been enrolled. If, at the end of stage 2 , fewer than or equal to 20 out of 68 patients had responded, this regimen was to be deemed not worthy of any further investigation in this patient population, unless clinical considerations suggest otherwise.

This sample size gives no less than a 0.738 chance of terminating enrollment early at the end of the first stage if the true $\mathrm{RR}$ is less than or equal to $22 \%$ (H0). This procedure provided a Type I error of 0.045 for testing of the null hypothesis that the RR is no greater than $22 \%$ and the statistical power is $90 \%$ when the RR is $40 \%$ (H1). All statistical tests were conducted at a two-sided $\alpha$ level of 0.05 , unless stated otherwise.

The primary analysis was completed using a 'qualified for clinical tumor response population', defined as females with histologic or cytologic diagnosis of advanced breast cancer previously treated with anthracyclines and taxanes, receiving no concurrent antitumor therapy, having presence of measurable disease as defined by RECIST, and receiving treatment with at least 1 dose of the study-drug of the assigned study regimen.

RR was defined as the sum of the number of patients with CR plus PR divided by the total number of qualified patients. Exact 95\% Pearson-Clopper confidence intervals (CIs) for the RR of each arm were calculated (39). RR was also analyzed by line of treatment (first-line vs. second-line) and visceral disease (yes vs. no).

Evaluation of time-to-event variables (secondary efficacy analyses) included quartiles estimated using the product limit method (40) and the corresponding 95\% CI based on the sign test (41). Safety variables were summarized descriptively.

For QoL assessment using the EORTC questionnaires QLQ-C30 and QLQ-BR23, observed values and absolute changes from baseline were summarized for patients who completed at least 1 questionnaire at baseline and at least 1 questionnaire after the first study-drug administration (a completed questionnaire was defined as one with at least
Table I. Baseline demographics and clinical characteristics of all enrolled patients.

\begin{tabular}{|c|c|c|}
\hline Variables & $\begin{array}{l}\text { Pemetrexed- } \\
\text { carboplatin } \\
\mathrm{N}=69, \mathrm{n}(\%)\end{array}$ & $\begin{array}{l}\text { Vinorelbine- } \\
\text { gemcitabine } \\
\mathrm{N}=66, \mathrm{n}(\%)\end{array}$ \\
\hline \multicolumn{3}{|l|}{ Age, years } \\
\hline Mean (SD) & $51.9(11.4)$ & $52.3(10.4)$ \\
\hline Median (min, max) & $52.0(29,75)$ & $51.5(30,77)$ \\
\hline \multicolumn{3}{|l|}{ Origin } \\
\hline Caucasian & $67(97.1)$ & $61(92.4)$ \\
\hline African & $1(1.4)$ & $3(4.5)$ \\
\hline Hispanic & $0(0.0)$ & $1(1.5)$ \\
\hline Asian & $1(1.4)$ & $1(1.5)$ \\
\hline \multicolumn{3}{|l|}{ ECOG PS } \\
\hline 0 & $39(56.5)$ & $39(59.1)$ \\
\hline 1 & $28(40.6)$ & $27(40.9)$ \\
\hline 2 & $2(2.9)$ & $0(0.0)$ \\
\hline \multicolumn{3}{|l|}{ Hormonal receptor status } \\
\hline $\mathrm{E}$ and $\mathrm{P}$ negative & $19(27.5)$ & $21(31.8)$ \\
\hline $\mathrm{E}$ and/or $\mathrm{P}$ positive & $49(71.0)$ & $44(66.7)$ \\
\hline Unknown & $1(1.4)$ & $1(1.5)$ \\
\hline \multicolumn{3}{|l|}{ HER-2/neutral assay } \\
\hline Positive & $12(17.4)$ & $13(19.7)$ \\
\hline Negative & $53(76.8)$ & $49(74.2)$ \\
\hline Not done/unknown & $4(5.8)$ & $4(6.1)$ \\
\hline \multicolumn{3}{|l|}{ Pathological diagnosis } \\
\hline Carcinoma, ductal & $64(92.8)$ & $54(81.8)$ \\
\hline Carcinoma, lobular & $4(5.8)$ & $5(7.6)$ \\
\hline Carcinoma, inflammatory & $1(1.4)$ & $3(4.5)$ \\
\hline Other & $0(0.0)$ & $4(6.1)$ \\
\hline Differentiation grade & $\mathrm{n}=63$ & $\mathrm{n}=60$ \\
\hline Grade I & $6(9.5)$ & $3(5.0)$ \\
\hline Grade II & $25(39.7)$ & $27(45.0)$ \\
\hline Grade III & $32(50.8)$ & $30(50.0)$ \\
\hline \multicolumn{3}{|l|}{ Line of treatment } \\
\hline 1st line & $21(30.4)$ & $19(28.8)$ \\
\hline 2nd line & 48 (69.6) & $47(71.2)$ \\
\hline \multicolumn{3}{|l|}{ Visceral disease } \\
\hline Yes & $55(79.7)$ & $56(84.8)$ \\
\hline No & $14(20.3)$ & $10(15.2)$ \\
\hline
\end{tabular}

ECOG PS, Eastern Cooperative Oncology Group Performance Status; $\mathrm{E}$, estrogen; P, progesterone; SD, standard deviation.

$50 \%$ of the questions answered). Analysis was performed according to the EORTC guidelines (http://www.eortc.be/ home/qol).

\section{Results}

Patient disposition, baseline demographics, and disease characteristics. A total of 135 patients, enrolled from June 2006 to April 2010 across 7 countries at 30 study centers, were randomly assigned with 69 patients in Arm A and 66 patients in Arm B. A total of 131 patients were treated (received at least 


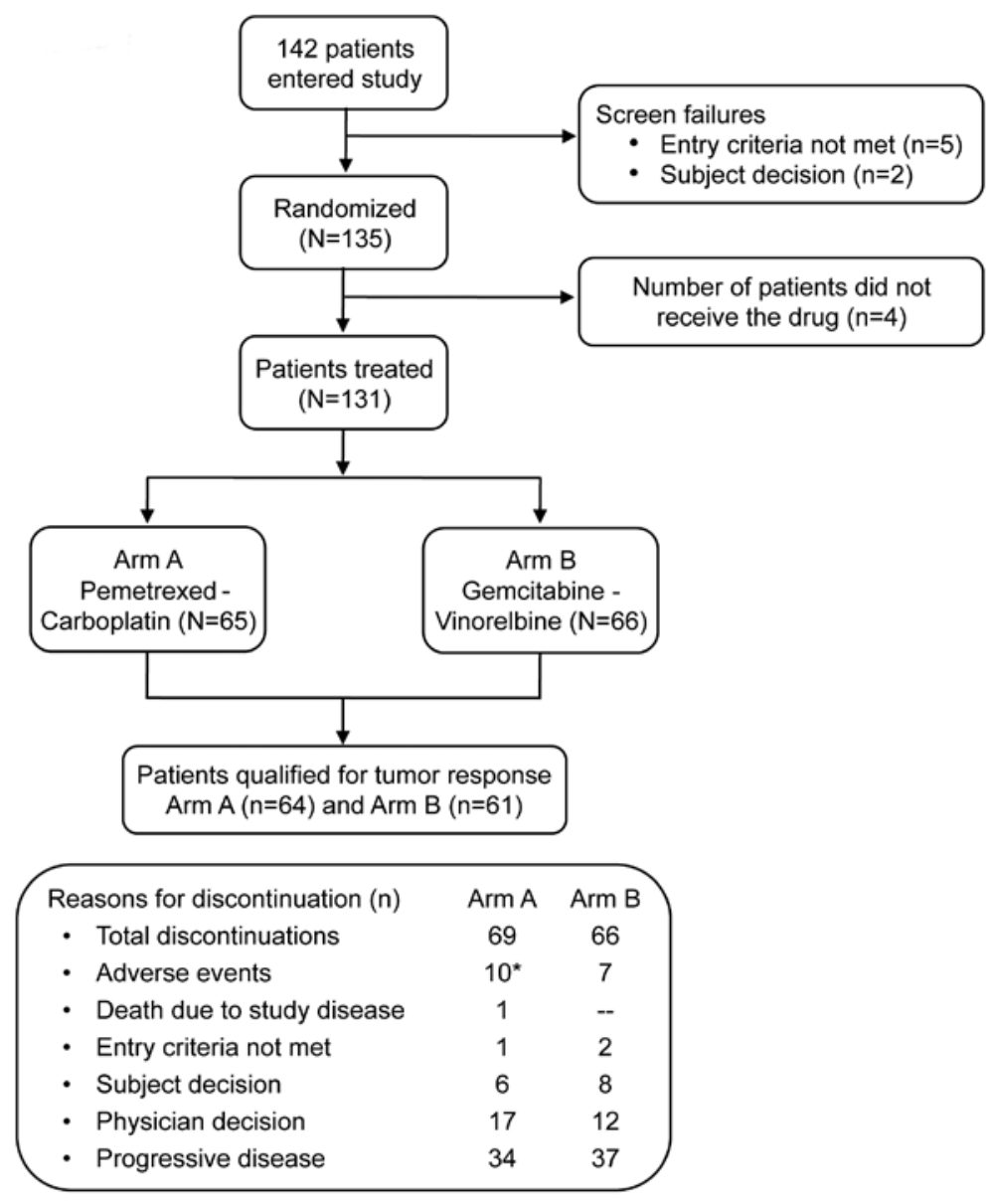

Figure 1. Patient disposition. "The 10 patients include 1 patient who discontinued due to AE but did not receive the study drug.

1 dose of study treatment), comprising 65 patients in Arm A and 66 patients in Arm B. The population qualifying for tumor response included 64 patients $(92.8 \%)$ in Arm A and 61 patients (92.4\%) in Arm B. Patient disposition is represented in Fig. 1.

The baseline demographics and disease characteristics of the study population are summarized in Table I. A majority of the patients were Caucasian (94.8\% overall) with mean age of 52 years in both treatment arms. Most of the patients had an ECOG PS of 0 (57.8\% overall), while 2 patients (2.9\%), both in Arm A, had an ECOG PS of 2. Most patients were receiving second-line treatment ( $70.4 \%$ overall) and more than $80 \%$ had visceral disease. The most common target lesion disease sites for the overall enrolled population were liver (45.5\%), lung (24.2\%) and lymph node (19.7\%).

Efficacy. The overall tumor response for the treatment arms is summarized in Table II. The population qualifying for tumor response included 64 patients $(92.8 \%)$ in Arm A and 61 patients (92.4\%) in Arm B. A RR of $26.6 \%$ (95\% CI: 16.3 , 39.1) was observed in Arm A and 29.5\% (95\% CI: 18.5, 42.6) was observed in Arm B at the end of stage 2 of the trial, which was lower than required to meet the primary endpoint (RR of more than 22\%). The PRs were similar in both treatment arms (26.6\%, 95\% CI: 16.3, 39.1 in Arm A and 26.2\%, 95\% CI: 15.8 , 39.1 in Arm B). There were 2 CRs and both were in Arm B (3.3\%, 95\% CI: 0.4, 11.3).
Among patients who qualified for tumor response, the first-line treatment RR was approximately $30 \%$ for both treatment arms [31.6\%, 95\% CI: 12.6, 56.6 in Arm A $(n=19)$ and $29.4 \%$, 95\% CI: $10.3,56.0$ in Arm B $(n=17)]$. Among patients receiving second-line treatment who qualified for tumor response, $\mathrm{RR}(95 \% \mathrm{CI})$ was $24.4 \%(12.9,39.5)$ in Arm A $(\mathrm{n}=45)$ and $29.5 \%(16.8,45.2)$ in Arm B $(n=44)$. In patients with visceral disease who qualified for tumor response, RR (95\% CI) was $23.5 \%$ [12.8, 37.5 in Arm A $(n=51)]$ and 32.1\% [19.9, 46.3 in Arm B ( $\mathrm{n}=53)]$. In patients with no visceral disease, RR was $38.5 \%$, 95\% CI: $13.9,68.4$ in Arm A $(n=13)$ and $12.5 \%, 95 \%$ CI: $0.3,52.7$ in $\operatorname{Arm~B~}(n=8)$. Analysis of tumor response using data for the enrolled population yielded results similar to the findings with the qualified population. The RR in this population was 17 [24.6\%, (95\% CI: 15.1, 36.5)] in Arm A and 19 [28.8\%, (95\% CI: 18.3, 41.3)] in Arm B.

All patients who showed a tumor response were included in analyses of DoR and TTR. TTPD and TTTF were presented for all treated patients. Secondary efficacy endpoints are summarized in Table III. The Kaplan-Meier curves for TTPD in the two treatment arms are presented in Fig. 2. The median TTPD was $5.1(95 \%$ CI: 4.1, 8.0) months for Arm A and $5.6(95 \%$ CI: 4.2, 7.5) months for Arm B (Table III).

Health outcomes. The population who qualified for QLQ-C30 and QLQ-BR23 analysis included 41 patients in Arm A and 39 


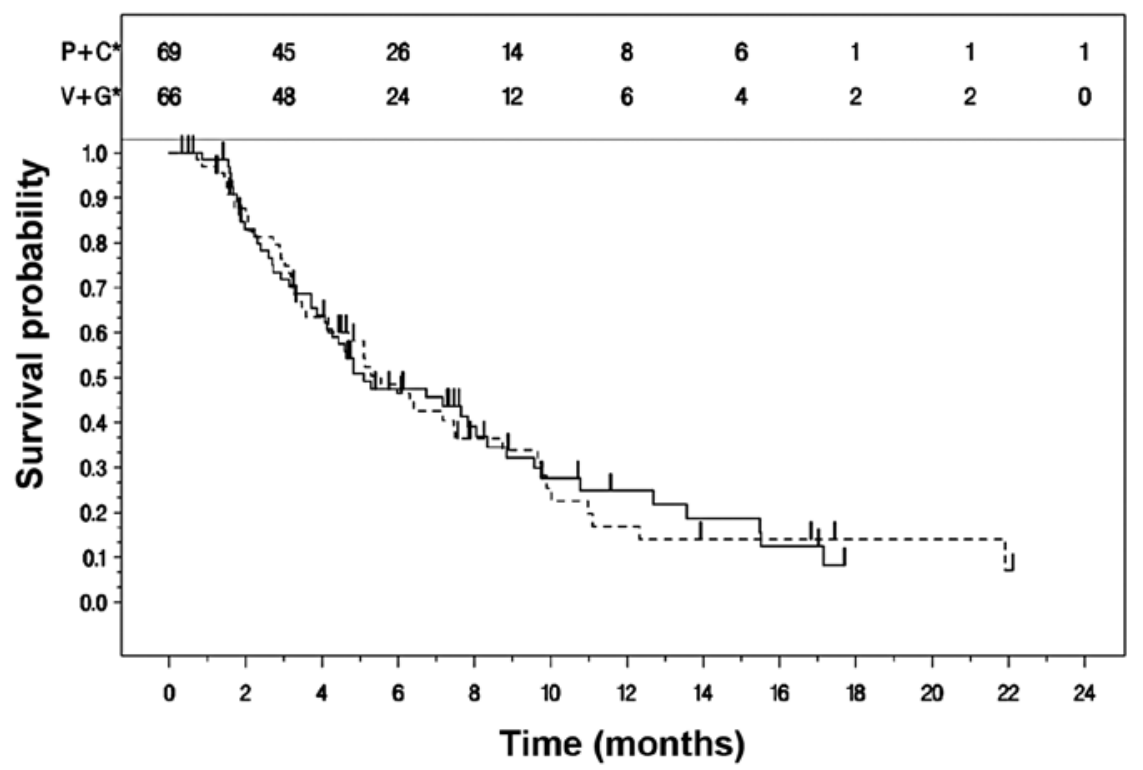

Figure 2. Kaplan-Meier curves for time to progressive disease in the two treatment groups. Solid line, pemetrexed-carboplatin (N=69, censored 21); dashed line, vinorelbine-gemcitabine ( $\mathrm{N}=66$, censored 20$)$. $\mathrm{P}+\mathrm{C}$, pemetrexed-carboplatin; $\mathrm{V}+\mathrm{G}$, vinorelbine-gemcitabine.

Table II. Best overall tumor response in patients who qualified for tumor response analyses.

\begin{tabular}{lcr}
\hline Parameters & $\begin{array}{c}\text { Pemetrexed-carboplatin } \\
\mathrm{N}=64, \mathrm{n}(\%),[95 \% \mathrm{CI}]\end{array}$ & $\begin{array}{c}\text { Vinorelbine-gemcitabine } \\
\mathrm{N}=61, \mathrm{n}(\%),[95 \% \mathrm{CI}]\end{array}$ \\
\hline Response rate (CR + PR, per RECIST criteria) & $17(26.6),[16.3,39.1]$ & $18(29.5),[18.5,42.6]$ \\
Best overall response rate & & \\
Complete response & $0(0.0),[0.0,5.6]$ & $2(3.3),[0.4,11.3]$ \\
Partial response & $17(26.6),[16.3,39.1]$ & $16(26.2),[15.8,39.1]$ \\
Stable disease & $23(35.9),[24.3,48.9]$ & $21(34.4),[22.7,47.7]$ \\
Disease progression & $17(26.6),[16.3,39.1]$ & $17(27.9),[17.1,40.8]$ \\
Unknown/not done & $7(10.9),[4.5,21.2]$ & $5(8.2),[2.7,18.1]$ \\
\hline
\end{tabular}

CI, confidence interval; CR, complete response; PR, partial response; RECIST, Response Evaluation Criteria in Solid Tumors.

Table III. Secondary efficacy endpoints, Kaplan-Meier estimates per treatment arm.

\begin{tabular}{lccccc}
\hline & \multicolumn{2}{c}{ Pemetrexed-carboplatin } & & \multicolumn{2}{c}{ Vinorelbine-gemcitabine } \\
\cline { 2 - 3 } Measure & $\begin{array}{c}\text { Patients with events } \\
\mathrm{n} / \mathrm{N}(\%)\end{array}$ & $\begin{array}{c}\text { Months, median } \\
{[95 \% \mathrm{CI}]}\end{array}$ & & $\begin{array}{c}\text { Patients with events } \\
\mathrm{n} / \mathrm{N}(\%)\end{array}$ & $\begin{array}{c}\text { Months, median } \\
{[95 \% \mathrm{CI}]}\end{array}$ \\
\hline Duration of response $^{\mathrm{a}}$ & $12 / 17(70.6)$ & $7.7[4.2,12.2]$ & & $16 / 19(84.2)$ & $7.5[4.9,8.3]$ \\
Time to response $^{\mathrm{a}}$ & $17 / 17(100)$ & $1.8[1.6,3.3]$ & & $19 / 19(100)$ & $1.8[1.6,3.1]$ \\
Time to progressive disease $^{\mathrm{b}}$ & $48 / 69(69.6)$ & $5.1[4.1,8.0]$ & & $46 / 66(69.7)$ & $5.6[4.2,7.5]$ \\
Time to treatment failure $^{\mathrm{b}}$ & $60 / 69(87.0)$ & $4.8[3.3,7.0]$ & & $58 / 66(87.9)$ & $5.1[3.5,6.3]$ \\
\hline
\end{tabular}

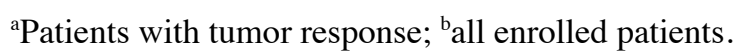

patients in Arm B for QLQ-C30, and 36 patients in Arm A and 38 patients in Arm B for QLQ-BR23 (Table IV). Baseline and change-from-baseline values at cycles 3 and 5 and at follow-up are presented in Table IV.
Safety. The median (range) number of cycles administered was similar for both treatment arms [6.0 (1,21) in Arm A and 6.0 $(1,20)$ in Arm B]. Exposure in the treatment arms as measured by relative dose intensity were: Arm A $[$ mean\% (SD) $]=88.5 \%$ 
Table IV. Health outcomes (EORTC QLQ-C30 global health status; QLQ-BR23 body image) in patients qualified for QLQ-C30 and QLQ-BR23 analyses.

\begin{tabular}{llccc}
\hline & \multicolumn{2}{l}{ Pemetrexed-carboplatin } & & \multirow{2}{*}{ Vinorelbine-gemcitabine } \\
\cline { 2 - 5 } Time point & $\mathrm{n}$ & Mean (SD) & $\mathrm{n}$ & Mean (SD) \\
\hline Baseline QLQ-C30 & 41 & $67.3(24.2)$ & 39 & $61.5(28.5)$ \\
Cycle 3, change from baseline & 35 & $-9(26.6)$ & 31 & $-4(21.8)$ \\
Cycle 5, change from baseline & 20 & $-10.4(28.3)$ & $-2.3(27.9)$ \\
Follow-up, change from baseline & 26 & $-21.5(32.5)$ & 22 & $-4.9(16.2)$ \\
Baseline QLQ-BR 23 & 36 & $77.5(30.5)$ & 38 & $75.4(24.1)$ \\
Cycle 3, change from baseline & 31 & $-3.6(23.5)$ & 31 & $-2.4(18.1)$ \\
Cycle 5, change from baseline & 18 & $-5.4(29.3)$ & $-3.9(13.1)$ \\
Follow-up, change from baseline & 24 & $-4.7(29.0)$ & $-3.7(20.4)$ \\
\hline
\end{tabular}

EORTC, European Organization for Research and Tumor of Cancer; QLQ-C30, Quality of Life Questionnaire; SD, standard deviation.

Table V. Grades 3 and 4 adverse events possibly related to studydrug that occurred in $\geq 10 \%$ of patients in each treatment group.

\begin{tabular}{lcc}
\hline $\begin{array}{l}\text { Adverse events } \\
\text { (laboratory and non-laboratory) }\end{array}$ & $\begin{array}{c}\text { Grade 3 } \\
\mathrm{n}(\%)\end{array}$ & $\begin{array}{c}\text { Grade 4 } \\
\mathrm{n}(\%)\end{array}$ \\
\hline $\begin{array}{l}\text { Pemetrexed-carboplatin, N=65 } \\
\text { Neutropenia }\end{array}$ & $15(23.1)$ & $9(13.8)$ \\
Thrombocytopenia & $9(13.8)$ & $6(9.2)$ \\
Anemia & $10(15.4)$ & $2(3.1)$ \\
Leukopenia & $9(13.8)$ & $1(1.5)$ \\
Vinorelbine-gemcitabine, N=66 & & \\
Neutropenia & $22(33.3)$ & $18(27.3)$ \\
Leukopenia & $9(13.6)$ & $2(3.0)$ \\
Fatigue & $8(12.1)$ & $1(1.5)$ \\
\hline
\end{tabular}

(12.7\%) for pemetrexed and 86.1\% (13.8\%) for carboplatin, and Arm B $=68.1 \%$ (14.4\%) for vinorelbine and 68.4\% (14.4\%) for gemcitabine. Over all cycles, day 8 dose reductions and omissions ranged from approximately 30 to $40 \%$ in Arm B compared with no omissions and approximately $20 \%$ of dose reductions in Arm A.

During study period or within the 30-day post-therapy period, 1 death in Arm A occurred due to the study disease and 1 death in Arm B occurred due to hepatic failure during follow-up period after 214 days of first dose. Nine patients (13.8\%) in Arm A and 7 patients $(10.6 \%)$ in Arm B (safety population) discontinued due to AEs. One additional patient who did not receive the drug in Arm A was also discontinued. The most common TEAE leading to discontinuation was neutropenia, reported in 1 patient $(1.5 \%)$ in Arm A and 3 patients (4.5\%) in Arm B. Two patients (3.1\%) in Arm A discontinued due to drug hypersensitivity and both were considered to be related to the study drug. Grade 3 congestive cardiac failure (SAE) leading to hospitalization was seen in 1 patient in Arm B, causing discontinuation.

At least $1 \mathrm{SAE}$ was reported in 18 patients $(27.7 \%)$ in Arm A and 22 patients (33.3\%) in Arm B; anemia, neutropenia and thrombocytopenia were the most commonly occurring in
Arm A and occurred in $>5 \%$ of the safety population. There were no drug-related SAEs that occurred in $>5 \%$ of patients in Arm B.

A summary of grades 3 and 4 TEAEs is provided in Table $\mathrm{V}$. Potentially drug-related CTCAE grade 3 or 4 TEAEs were seen in $36.9 \%$ of patients in Arm A and $60.6 \%$ of patients in Arm B, the most frequent being neutropenia.

\section{Discussion}

The present study was conducted to assess the antitumor activity and safety profiles of two chemotherapy regimens, pemetrexed-carboplatin and gemcitabine-vinorelbine in anthracycline- and taxane-pretreated patients with advanced breast cancer. The gemcitabine-vinorelbine combination has been studied extensively in various phase II trials and more recently in a phase III trial in pretreated advanced breast cancer patients, but the current study is the first to investigate the combination of pemetrexed-carboplatin in patients with advanced breast cancer pretreated with anthracycline and taxanes.

The RRs (95\% CI), the primary objective of this study, shown in both arms were moderate $[26.6 \%(16.3,39.1)$ in Arm A and $29.5 \%(18.5,42.6)$ in Arm B], since none met the predefined response at endpoint.

The RR shown by the gemcitabine-vinorelbine combination was comparable to previous studies (26,30-33,42-45). The more recent randomized phase III trial comparing the gemcitabinevinorelbine combination with capecetabine demonstrated an RR of $28.4 \%$ (27), which was comparable to the current study. The median TTPD and DoR in the present study were comparable with other phase II (TTPD, 5.7, 6.0 months; DoR, 6.9, 6.0 months) $(33,34)$ and phase III studies (TTPD, 5.4 months) $(27)$. Upon safety analysis, the rate of grades 3-4 neutropenia in this arm was found to be much higher $(60.6 \%)$ than that found in the previous studies (16.7-48.0\%) (30-33,42-45). However, no drugrelated SAEs were reported in $>5 \%$ patients in this treatment arm. In spite of the fact that there were higher rate of grade 3-4 neutropenia in this study, only two patients in Arm B had any infectious complications [1 patient $(1.5 \%)$ had staphylococcal sepsis and 1 patient $(1.5 \%)$ had streptococcal infection] and none were reported in Arm A. 
In an earlier study, where pemetrexed-carboplatin was given as first-line therapy, the RR was much higher (54\%) (13) compared to the present study. However, the RR of the combination in the current study was comparable to an earlier study (21\%) with pemetrexed alone in anthracycline-pretreated patients (8). Also, none of the patients achieved CR with the pemetrexed-carboplatin combination, either as a first-line or a second-line therapy. Both median TTPD (10.3 months) and DoR (11.1 months) were higher when the combination was used as a first-line treatment (13) than in the present study (TTPD, 5.1 months; DoR, 7.7 months). The rate of grade 3 and 4 neutropenia with pemetrexed-carboplatin was lower compared to first-line treatment studies with similar toxicity profiles (13). This could be attributed to the difference in the total durations of drug exposure in the earlier study compared to the present study ( 24 vs. 19 weeks). The high incidence of neutropenia despite full vitamin supplementation could be due to the additive myelosuppressive activity of the combination of carboplatin and pemetrexed (13).

The mean number of cycles administered was similar for both arms (Arm A, 6.3; Arm B, 6.2) but the exposure levels were lower in Arm B. The low dose intensity in Arm B appeared to be related to missed or reduced doses on day 8 ; over all cycles, day 8 dose reductions and omissions ranged from approximately 30 to $40 \%$ in Arm B, which might explain the relatively low exposure in Arm B.

One study limitation is that the majority (70\%) of patients enrolled in the present study had received two lines of previous treatment, which may have caused some study bias. Such heterogeneous population from prognosis point of view (1st and 2nd line) would explain the observed moderate RR. In addition, the non-comparative design of the $2 \mathrm{arms}$ of treatment prevented additional valuable conclusions.

From the above results it can be concluded that both combination therapies showed moderate efficacy and were well tolerated but further studies are still warranted. The results with pemetrexed and carboplatin were moderate but promising; results with gemcitabine and vinorelbine showed potential as in the previous studies. However, further research with newer drugs and newer combinations continues to be needed in order to deal with treatment failures as well as to arrest disease progression and palliate symptoms in patients with advanced breast cancer.

Clinical practice points. Screening and probably better treatments in early breast cancer decrease the numbers of metastatic breast cancer incidence. However, there is a selection of hard to treat population in this setting with the need to have non crossresistant drugs available. Most patients had anthracyclines and taxanes in the adjuvant setting. Therefore, well-tolerated active compounds are needed. Pemetrexed and carboplatin are both well-tolerated apart from the hematologic toxicities, which can easily be handled. The efficacy parameters are comparable to other well-tolerated combinations such as gemcitabine and vinorelbine and can be clinically accepted as an alternative treatment procedure. However, it must also be considered that pemetrexed is off-label in breast cancer treatment and the data are of limited value because these are phase II results. If platinum compounds will be further developed, particularly in basal or triple-negative breast cancer, and combination partners are needed, pemetrexed can be accepted as a promising compound.

\section{Acknowledgements}

This study was sponsored by Eli Lilly and Company, Indianapolis, IN, USA. The authors wish to thank the investigators, coordinators and patients for their participation in this study. V. Soldatenkova, V. Moreau and D. Desaiah and T. Bauknecht are employees of Eli Lilly and Company; M. Martin served on Lilly advisory boards and received remuneration; D. Amadori, E. Carrasco, S. Roesel, R. Labianca, B. Uziely and M. Martin served as investigators on this trial.

\section{References}

1. Ferlay J, Shin HR, Bray F, et al: Estimates of worldwide burden of cancer in 2008: GLOBOCAN 2008. Int J Cancer 127: 2893-2917, 2010

2. Jemal A, Bray F, Center MM, et al: Global Cancer Statistics. CA Cancer J Clin 61: 69-90, 2011.

3. Henderson IC, Berry DA, Demetri GD, et al: Improved outcomes from adding sequential Paclitaxel but not from escalating Doxorubicin dose in an adjuvant chemotherapy regimen for patients with node-positive primary breast cancer. J Clin Oncol 21: 976-983, 2003.

4. Hortobagyi GN: Treatment of breast cancer. N Engl J Med 339: 974-984, 1998.

5. Dean-Colomb W and Esteva FJ: Emerging agents in the treatment of anthracycline- and taxane-refractory metastatic breast cancer. Semin Oncol 35: S31-S38, 2008.

6. Overmoyer B: Options for the treatment of patients with taxanerefractory metastatic breast cancer. Clin Breast Cancer 8: S61-S70, 2008

7. Llombart-Cussac A, Theodoulou M, Rowland $\mathrm{K}$, et al: Pemetrexed in patients with locally advanced or metastatic breast cancer who had received previous anthracycline and taxane treatment: phase II study. Clin Breast Cancer 7: 380-385, 2006.

8. Martin M, Spielmann M, Namer M, et al: Phase II study of pemetrexed in breast cancer patients pretreated with anthracyclines. Ann Oncol 14: 1246-1252, 2003.

9. Miles DW, Smith IE, Coleman RE, et al: A phase II study of pemetrexed disodium (LY231514) in patients with locally recurrent or metastatic breast cancer. Eur J Cancer 37: 1366-1371, 2001.

10. Crown J and Pegram M: Platinum-taxane combinations in metastatic breast cancer: an evolving role in the era of molecularly targeted therapy. Breast Cancer Res Treat 79: S11-S18, 2003.

11. Martín M: Platinum compounds in the treatment of advanced breast cancer. Clin Breast Cancer 2: 190-208, 2001.

12. Perez EA, Hillman DW, Stella PJ, et al: A phase II study of paclitaxel plus carboplatin as first-line chemotherapy for women with metastatic breast carcinoma. Cancer 88: 124-131, 2000.

13. Garin A, Manikhas A, Biakhov M, et al: A phase II study of pemetrexed and carboplatin in patients with locally advanced or metastatic breast cancer. Breast Cancer Res Treat 110: 309-315, 2008

14. Heinemann V, Stemmler HJ, Wohlrab A, et al: High efficacy of gemcitabine and cisplatin in patients with predominantly anthracycline- and taxane-pretreated metastatic breast cancer. Cancer Chemother Pharmacol 57: 640-646, 2006.

15. Perez EA: Carboplatin in combination therapy for metastatic breast cancer. Oncologist 9: 518-527, 2004.

16. Brodowicz T, Kostler WJ, Möslinger R, et al: Single-agent gemcitabine as second- and third-line treatment in metastatic breast cancer. Breast 9: 338-342, 2000.

17. Spielmann M, Llombart-Cussac A, Kalla S, et al: Single-agent gemcitabine is active in previously treated metastatic breast cancer. Oncology 60: 303-307, 2001.

18. Blackstein M, Vogel CL, Ambinder R, et al: Gemcitabine as first-line therapy in patients with metastatic breast cancer: a phase II trial. Oncology 62: 2-8, 2002.

19. Heinemann V: Role of gemcitabine in the treatment of advanced and metastatic breast cancer. Oncology 64: 191-206, 2003.

20. Heinemann V: Gemcitabine in metastatic breast cancer. Expert Rev Anticancer Ther 5: 429-443, 2005. 
21. Modi S, Currie VE, Seidman AD, et al: A phase II trial of gemcitabine in patients with metastatic breast cancer previously treated with an anthracycline and taxane. Clin Breast Cancer 6: 55-60, 2005.

22. Albain KS, Nag SM, Calderillo-Ruiz G, et al: Gemcitabine plus Paclitaxel versus Paclitaxel monotherapy in patients with metastatic breast cancer and prior anthracycline treatment. J Clin Oncol 26: 3950-3957, 2008.

23. Seo JH, Oh SC, Choi CW, et al: Phase II study of a gemcitabine and cisplatin combination regimen in taxane resistant metastatic breast cancer. Cancer Chemother Pharmacol 59: 269-274, 2007.

24. Silvestris N, D'Aprile M, Andreola G, et al: Rationale for the use of gemcitabine in breast cancer (Review). Int J Oncol 24 389-398, 2004

25. Jones S, Winer E, Vogel C, et al: Randomized comparison of vinorelbine and melphalan in anthracycline-refractory advanced breast cancer. J Clin Oncol 13: 2567-2574, 1995.

26. Martín M, Ruiz A, Muñoz M, et al: Gemcitabine plus vinorelbine monotherapy in patients with metastatic breast cancer previously treated with anthracyclines and taxanes: final results of the phase III Spanish Breast Cancer Research Group (GEICAM) trial. Lancet Oncol 8: 219-225, 2007.

27. Pallis AG, Boukovinas I, Ardavanis A, et al: A multicenter randomized phase III trial of vinorelbine/gemcitabine doublet versus capecitabine monotherapy in anthracycline- and taxanepretreated women with metastatic breast cancer. Ann Oncol 23 $1164-1169,2012$

28. Degardin M, Bonneterre J, Hecquet B, et al: Vinorelbine (navelbine) as a salvage treatment for advanced breast cancer. Ann Oncol 5: 423-426, 1994.

29. Gregory RK and Smith IE: Vinorelbine - a clinical review. Br J Cancer 82: 1907-1913, 2000.

30. Haider K, Kornek GV, Kwasny W, et al: Treatment of advanced breast cancer with gemcitabine and vinorelbine plus human granulocyte colony-stimulating factor. Breast Cancer Res Treat 55: 203-211, 1999.

31. Valenza R, Leonardi V, Gebbia V, et al: Gemcitabine and vinorelbine in pretreated advanced breast cancer: a pilot study. Ann Oncol 11: 495-496, 2000.

32. Stathopoulos GP, Rigatos SK, Pergantas N, et al: Phase II trial of biweekly administration of vinorelbine and gemcitabine in pretreated advanced breast cancer. J Clin Oncol 20:37-41, 2002

33. Stemmler HJ, diGioia D, Freier W, Tessen, et al: Randomised phase II trial of gemcitabine plus vinorelbine vs gemcitabine plus cisplatin vs gemcitabine plus capecitabine in patients with pretreated metastatic breast cancer. Br J Cancer 104: 1071-1078, 2011.
34. Therasse P, Arbuck SG, Eisenhauer EA, et al: New guidelines to evaluate the response to treatment in solid tumors. European Organization for Research and Treatment of Cancer, National Cancer Institute of the United States, National Cancer Institute of Canada. J Natl Cancer Inst 92: 205-216, 2000.

35. Hughes A, Calvert P, Azzabi A, et al: Phase I clinical and pharmacokinetic Study of pemetrexed and carboplatin in patients with malignant pleural mesothelioma. J Clin Oncol 20: 3533-3544, 2002.

36. Cancer Therapy Evaluation Program. Common Terminology Criteria for Adverse Events, Version 3.0, 2003. National Cancer Institute Available, Published online August 9, 2006; available at: http://ctep.cancer.gov/protocolDevelopment/electronic_ applications/docs/ctcev3.pdf. Accessed November 14, 2011.

37. Nagel GC, Schmidt S, Strauss BM, et al: Quality of life in breast cancer patients: a cluster analytic approach. Empirically derived subgroups of the EORTC-QLQ BR 23 - a clinically oriented assessment. Breast Cancer Res Treat 68: 75-87, 2001.

38. Simon R: Optimal two-stage designs for phase II clinical trials. Control Clin Trials 10: 1-10, 1989.

39. Clopper CJ and Pearson ES: The use of confidence or fiducial limits illustrated in the case of the binomial. Biometrika 26: 404-413, 1934.

40. Kaplan EL and Meier P: Nonparametric estimation from incomplete observations. J Am Stat Assoc 53: 457-481, 1958.

41. Brookmeyer R and Crowley J: A confidence interval for the median survival time. Biometrics 38: 29-41, 1982.

42. Nicolaides C, Dimopoulos MA, Samantas E, et al: Gemcitabine and vinorelbine as second-line treatment in patients with metastatic breast cancer progressing after first-line taxane-based chemotherapy: a phase II study conducted by the Hellenic Cooperative Oncology Group. Ann Oncol 11: 873-875, 2000.

43. Park IH, Ro J, Lee KS, et al: Phase II study of gemcitabine in combination with vinorelbine versus gemcitabine followed by vinorelbine for metastatic breast cancer. Invest New Drugs 28: 659-669, 2010

44. Shehata S, Saad E, Goda Y, et al: A phase II study of gemcitabine combined with vinorelbine as first-line chemotherapy for metastatic breast cancer. Hematol Oncol Stem Cell Ther 3: 1-6, 2010.

45. Morabito A, Filippelli G, Palmeri S, et al: The combination of gemcitabine and vinorelbine is an active regimen as secondline therapy in patients with metastatic breast cancer pretreated with taxanes and/or anthracyclines: a phase I-II study. Breast Cancer Res Treat 78: 29-36, 2003. 